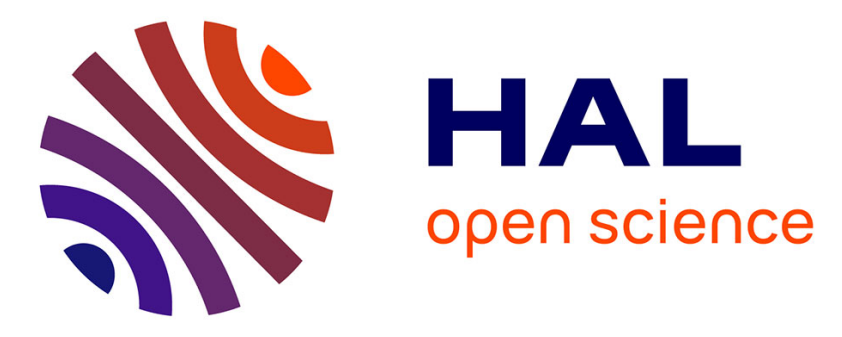

\title{
Polynomial superlevel set approximation of swept-volume for computing collision probability in space encounters
}

Denis Arzelier, Florent Bréhard, Mioara Joldeş, Jean-Bernard Lasserre, Sohie Laurens, Aude Rondepierre

\section{To cite this version:}

Denis Arzelier, Florent Bréhard, Mioara Joldeş, Jean-Bernard Lasserre, Sohie Laurens, et al.. Polynomial superlevel set approximation of swept-volume for computing collision probability in space encounters. 60th IEEE Conference on Decision and Control (CDC 2021), IEEE, Dec 2021, Austin, Texas, United States. 10.1109/CDC45484.2021.9683445 . hal-03158347

\section{HAL Id: hal-03158347 \\ https: / hal.laas.fr/hal-03158347}

Submitted on 3 Mar 2021

HAL is a multi-disciplinary open access archive for the deposit and dissemination of scientific research documents, whether they are published or not. The documents may come from teaching and research institutions in France or abroad, or from public or private research centers.
L'archive ouverte pluridisciplinaire HAL, est destinée au dépôt et à la diffusion de documents scientifiques de niveau recherche, publiés ou non, émanant des établissements d'enseignement et de recherche français ou étrangers, des laboratoires publics ou privés. 


\title{
Polynomial superlevel set approximation of swept-volume for computing collision probability in space encounters
}

\author{
D. Arzelier, F. Bréhard, M. Joldeş, J.-B. Lasserre, S. Laurens, A. Rondepierre
}

\begin{abstract}
Computing long-term collision probability in space encounters is usually based on integration of a multivariate Gaussian distribution over the volume of initial conditions which generate collisions in the considered time interval. As this collision set is very difficult to determine analytically, for practical computation various simplifications are made in the literature. We present a new method for computing the collision probability based on two steps. Firstly, a higher-order outer-approximation of the swept-volume by a polynomial superlevel set is obtained as an optimal solution of a polynomial optimization problem. This has the advantage of providing approximate closed-form descriptions of the collision-prone states which can then be effectively used for long-term and repeated conjunctions analysis. From a computational viewpoint, one has to solve a hierarchy of linear matrix inequality problems of increasing size, which provide approximations (i) of increasing accuracy and (ii) convergent in volume to the original set. Secondly, once such a polynomial representation is computed, a high-order quadrature scheme for volumes implicitly defined by a polynomial superlevel sets is employed. Finally, the method is illustrated on some numerical examples borrowed from the literature.
\end{abstract}

\section{INTRODUCTION}

Since the collision between the Russian satellite COSMOS 1934 and one debris of COSMOS 926 in December 1991, no less than eight orbital collisions have been reported between operational satellites. Space agencies and operators of the field have established alert procedures to assess the risks of collision for controlled satellites, and to authorize avoidance maneuvers if the predicted risk, measured by a probability of collision, exceeds some tolerance threshold. Risk evaluation is

This work was supported by CNES

D. Arzelier is with ROC team, LAAS-CNRS, 7, avenue du Colonel Roche 31031 Toulouse, France denis.arzelierdlaas.fr

F. Bréhard is with CFHP research group, CRIStAL laboratory, CNRS, Université de Lille - Campus scientifique, Avenue Henri Poincaré, 59655 Villeneuve d'Ascq, France florent.brehardeuniv-lille.fr

M. Joldes is with ROC team, LAAS-CNRS, 7, avenue du Colonel Roche 31031 Toulouse, France joldes@laas. fr

J.B. Lasserre is with MAC team, LAAS-CNRS, 7, avenue du Colonel Roche 31031 Toulouse, France jean-bernard.lasserre@laas.fr

S. Laurens is with Centre National d'Etudes Spatiales (CNES), 18 Avenue Edouard Belin, 31400 Toulouse, France sophie.laurensecnes.fr

A. Rondepierre is with ROC team, LAAS-CNRS, 7, avenue du Colonel Roche 31031 Toulouse, France aude.rondepierredinsa-toulouse.fr also performed after an avoidance maneuver to assess its benefit. When assessing conjunctions between two objects, the information usually available is: (1) a bound on the radius of the involved objects assumed to be spherical; (2) normal probability distributions (mean and covariance values) of the state vectors of the objects at the Time of Closest Approach (TCA, when their nominal relative distance is estimated to be minimal).

In this context, two classes of encounters are defined. For the so-called short-term encounters [9], [1], [4], [21], the objects' relative velocity is assumed to be very high (several $\mathrm{km} / \mathrm{s}$ ), so that the relative motion is considered rectilinear on the encounter time interval. When the cross-correlations between the estimated states of the two objects, as well as their velocity uncertainty are neglected, the formulation of the collision probability is greatly simplified. In brief, the relative positions which generate collisions lie in a three-dimensional cylinder (also called collision tube), on which a Gaussian density is to be integrated. Finally, this reduces to computing a two-dimensional Gaussian integral on a disk.

In the second class, called long-term encounters [4], [7], the relative velocity is of the order of $\mathrm{m} / \mathrm{s}$ and both objects spend a significant amount of time in proximity to each other. This type of encounter is more common in the context of formation flying or proximity operations. Computing collision probabilities, in this so-called nonlinear framework, is considerably more difficult than for the short-term case. Even though the state distribution at TCA is a Gaussian one, the integration volume is no-longer a cylinder and can be very intricate (see Section III for details). Due to this, generalization attempts were proposed for specific cases of configurations [18], [16], [4], but these approaches are relatively limited because of their characterization for particular relative trajectories.

In [7], V. Coppola proposes a different mathematical formalization and generalization. Roughly speaking, using a change of variable, the complicated integration volume at TCA is mapped via the dynamics at each time on the so-called hard-body sphere (which is a three-dimensional sphere of known radius equal to sum of the objects radii). In turn, this implies propagating the TCA distribution (both position and velocity) via the non-rectilinear dynamics. This change of variable 
is correct when imposing for each relative trajectory at most one entry crossing the hard-body sphere, which means that multiple encounters between the two objects are excluded. To tackle a practical implementation, the propagated distribution is assumed to remain Gaussian during the encounter interval. These two assumptions provide a rather restricted framework, which is however currently one of the most accomplished in the literature. Another approach, sketched in the works of Chan [5], [6], consists in focusing on a different mathematical description of the integration volume at TCA, which is also called the swept-volume. Generated by the propagation of the hard-body during the encounter duration, it is defined as a union of ellipsoids (see Section III-A) and numerically characterized by its envelope in the 3dimensional case, or in an ad-hoc manner, with various trivial simplifications for lower dimensional cases. However, a general method relying on both (i) an effective characterization of the swept-volume (when its shape is not trivially reduced to a cylinder), and (ii) computation of the subsequent integral of the Gaussian density over such a volume, is missing in the literature.

Following up this intuition, in this article we invoke polynomial optimization to provide approximate closedform descriptions of the collision-prone states, which can be effectively used for long-term and repeated conjunctions. This is a generalization with respect to Coppola's formulation which cannot handle multiple conjunctions correctly. Actually, even a visual accurate outer-approximation of the swept volume can provide important insights on the practical type of encounter. For instance, a straight cylinder form can confirm some of the encounter assumptions of the short-term framework. The proposed method is based on two steps: (1) higherorder implicit 11 outer-approximation of the swept-volume by a Polynomial Superlevel Set (PSS). From a computational viewpoint, one has to solve a hierarchy of linear matrix inequality problems (each providing an outerapproximation of increasing accuracy) with convergence in volume to the original set. (2) Once such a polynomial representation has been computed, a high-order quadrature scheme for volumes implicitly defined by a PSS is employed. With PSS approximation, highly non-convex shapes can be outer-approximated accurately, which in turn allows for further analysis of so-called longterm encounters. The method is illustrated on numerical examples borrowed from the literature.

\section{ENCOUNTER MODELING AND PROBLEM STATEMENT}

Consider an operational spacecraft (called primary and denoted by $p$ ) in orbit around the Earth and a space

\footnotetext{
${ }^{1}$ Implicit means defined by an inequality constraint, as opposed to an explicit parametric representation of each point of the set.
}

debris (called secondary and denoted by $s$ ). Their state is described by their position and velocity vectors $r_{\star}$ and $v_{\star}$, in a reference frame $\hat{\mathcal{R}}(\star=p$ or $\star=s)$. Classically the objects are modeled as spheres [1], [18], [4], [7], of known radii $R_{\star}$, since this allows for factoring out their orientation (attitude) and, for a conservative modeling of the secondary object, whose geometry is often poorly known (see Figure 1). With this assumption, a collision occurs when the relative distance between $r_{p}$ and $r_{s}$ is less than the so-called hard-body radius $R=R_{p}+$ $R_{s}$. Based on this notion, it is natural to focus on the

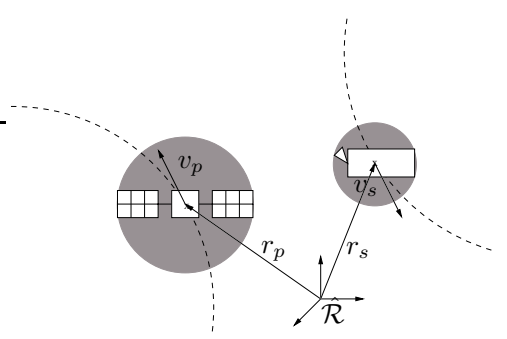

Fig. 1: Encounter between two spherical objects.

relative state vector $x_{r}^{T}=\left(\left(r_{s}-r_{p}\right)^{T},\left(v_{s}-v_{p}\right)^{T}\right) \in$ $\mathbb{R}^{6}$, whose dynamics are given by:

$$
\left\{\begin{aligned}
\dot{x}_{r}(t) & =f\left(t, x_{r}(t)\right), \quad t \in\left[t_{0}, t_{f}\right] \\
x_{r}\left(t_{0}\right) & =x_{r}^{0}
\end{aligned}\right.
$$

where $f$ is a real Lipschitz continuous vector field and $\mathcal{T}:=\left[t_{0}, t_{f}\right]$ is the given time interval of the encounter. In general, these equations include the Newtonian gravitational central field and possible orbital perturbations (non spherical Earth, atmospheric drag, e.g.). It is assumed that, for each given relative initial condition $x_{r}^{0} \in \mathbb{R}^{6}$, the solution $x_{r}\left(t \mid x_{r}^{0}\right)$ (also called trajectory or sample path) of the system (1) exists and is unique for $t \in \mathcal{T}$.

The initial conditions $x_{r}^{0} \in \mathbb{R}^{6}$ are usually subject to uncertainties, and so, they are supposed to be distributed according to a given probability measure $\mu_{I}$, with density $\rho_{I}$. As mentioned in the introduction, in practice the uncertainty distribution is usually given and estimated to be Gaussian at $t_{T C A} \in \mathcal{T}$. In the following, without loss of generality, we suppose that $t_{T C A}=t_{0}$ (the proposed algorithms are easily tunable for the case $t_{0}<t_{T C A}<t_{f}$, as illustrated by examples in Sec. VI). Assumption 1 (Initial Gaussian distribution): The relative initial conditions are normally distributed:

$$
\rho_{I}\left(x_{r}^{0}\right):=\frac{e^{-\frac{1}{2}\left(x_{r}^{0}-m_{I}\right)^{T} P_{I}^{-1}\left(x_{r}^{0}-m_{I}\right)}}{(2 \pi)^{3} \sqrt{\operatorname{det}\left(P_{I}\right)}},
$$

with mean relative vector $m_{I}$ and covariance matrix $P_{I}$. In relative dynamics, the notion of collision transcribes to a relative trajectory entering a forbidden region $\mathcal{X}_{R}$ :

$$
\mathcal{X}_{R}=\left\{x_{r}^{T}=\left(r_{r}^{T}, v_{r}^{T}\right) \in \mathbb{R}^{6} \mid\left\|r_{r}\right\|_{2}^{2}-R^{2} \leqslant 0\right\} .
$$


Definition 1 (Collision): Given a relative initial condition $x_{r}^{0} \in \mathbb{R}^{6}$, a time interval $\mathcal{T}$ and a forbidden region $\mathcal{X}_{R}$, a collision occurs if there exists $t \in \mathcal{T}$ such that $x_{r}\left(t \mid x_{r}^{0}\right) \in \mathcal{X}_{R}$.

Definition 2 (Collision domain/swept-volume): The domain of collision $\mathcal{X}_{\mathcal{T}}^{0}$ is the set of relative initial conditions leading to collision on the time interval $\mathcal{T}$, namely:

$$
\mathcal{X}_{\mathcal{T}}^{0}=\left\{x_{r}^{0} \in \mathbb{R}^{6} \mid \exists t \in \mathcal{T}, x_{r}\left(t \mid x_{r}^{0}\right) \in \mathcal{X}_{R}\right\} .
$$

The problem of computing the collision probability is formally stated as:

Problem 1 (General formulation): Let the dynamics in (1), a time interval $\mathcal{T}$ and a forbidden region $\mathcal{X}_{R}$. Provided that the initial conditions $x_{r}^{0} \in \mathbb{R}^{6}$ are distributed according to a given probability measure $\mu_{I}$, compute the probability that a collision occurs:

$$
\mathcal{P}_{c}(\mathcal{T}):=\mathbb{P}\left(x_{r}^{0} \in \mathcal{X}_{\mathcal{T}}^{0}\right)=\mu_{I}\left(\mathcal{X}_{\mathcal{T}}^{0}\right)=\int_{\mathcal{X}_{\mathcal{T}}^{0}} \mathrm{~d} \mu_{I}
$$

Note that with Assumption 11 Eq. (5) becomes:

$$
\mathcal{P}_{c}(\mathcal{T})=\int_{\mathcal{X}_{\mathcal{T}}^{0}} \rho_{I}\left(x_{r}\right) \mathrm{d} x_{r}
$$

Problem 1 is in general very difficult. A first issue is to determine the domain of integration, which strongly depends on the chosen model for the relative dynamics [4]. A theoretical solution was proposed for a polynomial vector field $f$, in the framework of moments-measures, sum-of-squares (SOS) in a series of works [23], [11], [3]: using Liouville's equation, the nonlinear dynamics is lifted into a linear equation on measures, which is then solved using the Lasserre moment-SOS hierarchy of relaxations. However, in addition to the inherent numerical complexity of the present high-dimensional situation, we have also encountered numerical issues. This led us to consider other numerically-tractable solutions with a more practical utility, as discussed in what follows.

\section{SWEPT-VOLUME AS A UNION OF BASIC SEMI-ALGEBRAIC SETS}

The main goal is to obtain a more tractable characterization of the integration domain $\mathcal{X}_{\mathcal{T}}^{0}$. From Equation (4), one obtains a description of $\mathcal{X}_{\mathcal{T}}^{0}$ as a union of sets, by retro-propagating with the inverse flow $\varphi_{t}^{t_{0}}$ of the relative dynamics the set $\mathcal{X}_{R}$ at each time $t \in \mathcal{T}$ :

$$
\mathcal{X}_{\mathcal{T}}^{0}=\bigcup_{t \in \mathcal{T}}\left\{\varphi_{t}^{t_{0}}\left(x_{r}\right) \mid x_{r} \in \mathcal{X}_{R}\right\}
$$

However, due to the complicated nature of the inverse flow (no closed-form solution in general), this set can hardly be analytically described, so various additional assumptions have been made in the literature [4], [7], [5]. In this work, the assumption of linearized relative dynamics is employed, which is quite commonly used for uncertainty propagation in such applications [2] (see Sec. VI for a specific example of linearization).

Assumption 2 (Linearized relative dynamics): The relative dynamics flow is linear and invertible. The solution of the relative dynamics equation is therefore known via a given state transition matrix $\Phi\left(\cdot, t_{0}\right): \mathcal{T} \rightarrow \mathbb{R}^{6}$ :

$$
x_{r}\left(t \mid x_{r}^{0}\right)=\Phi\left(t, t_{0}\right) x_{r}^{0}, \text { for } t \in \mathcal{T} .
$$

From Equations (8) and (4), the swept-volume becomes:

$$
\begin{aligned}
& \mathcal{X}_{\mathcal{T}}^{0}=\left\{x_{r}^{0} \in \mathbb{R}^{6}: \exists t \in \mathcal{T},\right. \\
& \left.R^{2}-x_{r}^{0^{T}} \Phi\left(t, t_{0}\right)^{T} I_{11} \Phi\left(t, t_{0}\right) x_{r}^{0} \geqslant 0\right\},
\end{aligned}
$$

where the matrix $I_{11} \in \mathbb{R}^{6 \times 6}$ is defined by $I_{11}:=$ $\left(\begin{array}{rr}I_{3} & 0 \\ 0 & 0\end{array}\right)$. This appears in the formula simply because only the positions (first 3 coordinates of $x_{r}\left(t \mid x_{r}^{0}\right)$ ) are constrained to belong to $\mathcal{X}_{R}$. An observation made in [5] is that when the last 3 coordinates of $x_{r}^{0}$ are fixed (no velocity uncertainty), the above inequality describes an ellipsoid for each time $t$. Since this simplification is important in practice, we detail it in what follows.

\section{A. No velocity uncertainty}

Denote the initial state vector $x_{r}^{0} \in \mathbb{R}^{6}$ by $x_{r}^{0^{T}}:=$ $\left(r_{r}^{0^{T}}, v_{r}^{0^{T}}\right)$, where the relative velocity $v_{r}^{0} \in \mathbb{R}^{3}$ is exactly known (not a random vector). Firstly, note that the integral in Equation (6) becomes three-dimensional. Secondly, we are only interested in relative positions in the swept-volume $\mathcal{X}_{\mathcal{T}}^{0}$. Straightforward calculations lead to the following characterization.

Proposition 1 (3D Swept-volume): Let the relative dynamics transition matrix $\Phi\left(t, t_{0}\right)$ be given and denoted by blocks by $\Phi:=\left(\begin{array}{cc}\Phi_{11} & \Phi_{12} \\ \Phi_{21} & \Phi_{22}\end{array}\right)$.

Assume that $\Phi_{11}\left(t, t_{0}\right)$ is invertible for each $t \in \mathcal{T}$. The swept-volume $\mathcal{X}_{r \mathcal{T}}^{0} \in \mathbb{R}^{3}$, containing all the relative positions $r_{r}^{0} \in \mathbb{R}^{3}$ which lead to collisions during the time interval $\mathcal{T}$, is described by a union of ellipsoids,

$$
\mathcal{X}_{r \mathcal{T}}^{0}=\bigcup_{t \in \mathcal{T}} \mathcal{E}_{t, t_{0}}
$$

with

$$
\begin{aligned}
& \mathcal{E}_{t, t_{0}}:=\left\{r_{r}^{0} \in \mathbb{R}^{3}:\right. \\
& \left.R^{2}-\left(r_{r}^{0}-c\left(t, t_{0}\right)\right)^{T} Q\left(t, t_{0}\right)^{-1}\left(r_{r}^{0}-c\left(t, t_{0}\right)\right) \geqslant 0\right\},
\end{aligned}
$$

where

$$
\begin{aligned}
& c\left(t, t_{0}\right)=-\Phi_{11}\left(t, t_{0}\right)^{-1} \Phi_{12}\left(t, t_{0}\right) v_{r}^{0} \\
& Q\left(t, t_{0}\right)=\Phi_{11}\left(t, t_{0}\right)^{-1} \Phi_{11}\left(t, t_{0}\right)^{-T}
\end{aligned}
$$

Remark 1 (The 3D swept-volume as a compact set):

Provided that the matrix $\Phi_{11}\left(t, t_{0}\right)$ is invertible for each $t \in \mathcal{T}$, each ellipsoid $\mathcal{E}_{t, t_{0}}$ is proper i.e., $Q\left(t, t_{0}\right)$ has full rank, and thus their union is compact. This is important for practical implementations which are 
developed in Sec. IV-C. Otherwise, the swept-volume can still be described by the union of non-necessary proper 3D quadratic forms.

\section{B. Gaussian integral of union of semi-algebraic sets}

Motivated by the previous description of the sweptvolume as a union of ellipsoids, one observes that in the general case, by taking a sufficiently fine discretization of size $N, \tau_{N}:=\left\{t_{0} \leqslant \cdots t_{i} \leqslant \cdots \leqslant t_{f}\right\}$, the constraints describing subsets $\mathcal{K}_{i} \subseteq \mathcal{X}_{\mathcal{T}}^{0}$ :

$$
\mathcal{K}_{i}:=\left\{x_{r}^{0} \in \mathbb{R}^{6}: R^{2}-x_{r}^{0^{T}} \Phi\left(t_{i}, t_{0}\right)^{T} I_{11} \Phi\left(t_{i}, t_{0}\right) x_{r}^{0} \geqslant 0\right\},
$$

provide an approximate description of $\mathcal{X}_{\mathcal{T}}^{0}$ as a union of basic semi-algebraic sets (which are neither disjoint, nor compact in general):

$$
\mathcal{K}:=\bigcup_{i=1}^{N} \mathcal{K}_{i} \subseteq \mathcal{X}_{\mathcal{T}}^{0} .
$$

The main advantage of expressing the swept-volume as a union of basic semi-algebraic sets, is that it can be exploited in the framework of Polynomial Optimization [12]. The simplified formulation of $\mathrm{Pb}$. 1 reads:

Problem 2 (Integration on union of semi-algebraic sets): Given a union of basic semi-algebraic sets as in Eq. (11), 10), and a Gaussian probability measure $\mu_{I}$ compute the integral:

$$
\tilde{\mathcal{P}}_{c}(\mathcal{T}):=\mu_{I}(\mathcal{K})=\int_{\mathcal{K}} \mathrm{d} \mu_{I} .
$$

This problem has a theoretical interest of its own and has been already addressed in the literature [14] based on the measure-moments framework. In brief, it can be proven that Problem (2) is equivalent to an infinite-dimensional linear program on positive measures.

Firstly, let us fix some necessary notations. Given a Borel set $\Omega \subset \mathbb{R}^{n}$, let $\mathcal{M}(\Omega)$ be the Banach space of finite signed Borel measures on $\Omega$, equipped with the total variation norm. For $\mu, \nu \in \mathcal{M}(\Omega)$, denote by $\operatorname{supp}(\mu)$ the support of $\mu$ (i.e. the smallest closed set $\Gamma$ of $\Omega$ s.t. $|\mu|(\Omega \backslash \Gamma)=0$ ); denote $\mu \leqslant \nu$ when $\mu(A) \leqslant \nu(A)$ for any measurable set $A \subseteq \Omega$; finally, a positive measure $\mu$ is denoted by $\mu \geqslant 0$.

Proposition 2 (Thm. 3 [14]): The following problem has an optimal solution and $\bar{\kappa}_{\mathcal{K}}^{*}=\tilde{\mathcal{P}}_{c}(\mathcal{T})$.

$$
\begin{aligned}
\bar{\kappa}_{\mathcal{K}}^{*}=\quad & \sup _{\mu_{1}, \ldots, \mu_{N} \in \mathcal{M}\left(\mathbb{R}^{6}\right)} \sum_{i=1}^{N} \mu_{i}\left(\mathcal{K}_{i}\right), \\
& \sum_{i=1}^{N} \mu_{i} \leqslant \mu_{I}, \\
\text { s.t. } & \operatorname{supp}\left(\mu_{i}\right) \subseteq \mathcal{K}_{i}, i=1, \ldots, N \\
& \mu_{i} \geqslant 0, i=1, \ldots, N .
\end{aligned}
$$

Roughly speaking, this problem aims at maximizing the sum of masses of $N$ positive measures $\mu_{i}$ (with $\left.\operatorname{supp}\left(\mu_{i}\right) \subseteq \mathcal{K}_{i}\right)$ whose sum is dominated by the known Gaussian measure $\mu_{I}$. Problem (13) is numerically solved via a monotone sequence of upper bounds converging to the optimal value of (13). Each upper bound is obtained by solving a truncated-moment problem similar to the one applied in [10] and [13] for the computation of respectively Lebesgue or Gaussian measures of a basic semi-algebraic set. The monotonic convergence, which is rather slow in general, may be significantly improved using a technique based on Stokes' formula. This requires to use a polynomial which vanishes on the algebraic boundary of the considered set [14]. Unfortunately this acceleration technique cannot be directly used here, due to the high number of sets $\mathcal{K}_{i}$ involved. Indeed a polynomial that vanishes on the boundary of the union can have a potential very high degree, which results in intractable additional Stokes' constraints. Another limitation of directly solving (13) is that when its optimal value is very small, very high order relaxations are needed. The latter have a large size and are numerically ill-conditioned because of high values of some moments of the Gaussian to be computed.

Hence, motivated also by the practical question of obtaining a closed-form representation of the sweptvolume, we chose instead another similar approach for Problem 2, with two main steps: (1) Find an implicit representation of the integration domain $\mathcal{K}$ by a PSS [8], [14]; (2) Compute the integral (12) with a high-order quadrature for volumes implicitly defined by a PSS [20].

\section{PSS APPROXIMATIONS OF THE SWEPT-VOLUME}

It was shown in [8] (see also [10], [14] for similar works) that a union $\overline{\mathcal{K}}=\bigcup_{i=1}^{N} \overline{\mathcal{K}}_{i} \subseteq \mathbb{R}^{n}$, of compact basic semialgebraic sets $\overline{\mathcal{K}}_{i}$ (given by a conjunction of polynomial inequalities), can be efficiently approximated by a PSS. Let $\mathbb{R}[x]_{d}$ be the vector space of polynomials in the variables $x=\left(x_{1}, \ldots, x_{n}\right)$ over reals, of total degree at most $d$. Moreover, suppose that $\overline{\mathcal{K}}$ can be outerbounded by a hyper-rectangle $\overline{\mathcal{K}} \subseteq \mathcal{B}:=[a, b]=$ $\left\{x \in \mathbb{R}^{n}, a_{i} \leqslant x_{i} \leqslant b_{i}\right.$, for $\left.i=1, \ldots, n\right\}, a, b \in \mathbb{R}^{n}$.

Definition 3 (PSS): A degree $d$-PSS approximation for $\overline{\mathcal{K}}$ is defined by a polynomial $p_{d} \in \mathbb{R}[x]_{d}$, s.t.

$$
\overline{\mathcal{K}} \subseteq \operatorname{PSS}_{p_{d}}:=\left\{x \in \mathcal{B}: p_{d}(x) \geqslant 1\right\} .
$$

A first technical issue is that the swept-volume in Eq. (11) is not compact in general. An exception is the 3D case (no velocity uncertainty, cf. Remark 1) for which the method of [8] can be directly applied.

For the 6D general case, a straightforward solution is to rely on the fact that the approximation computed for $\mathcal{K}$ is to be used afterwards for integrating the multivariate Gaussian density (12). Hence, one can consider a suitable $h-\sigma$ ellipsoid corresponding to the given covariance matrix $P_{I}$ (say $h=8.5$ in practice, to be tuned depending on numerical requirements) and bound it by a 
hyper-rectangle $\mathcal{B}$. Then, one uses the sets $\overline{\mathcal{K}}_{i}=\mathcal{K}_{i} \cap \mathcal{B}$, which are compact. With this additional approximation, we next proceed with a description of the method and algorithms adapted from [8], [14] to our case, to obtain a PSS of fixed degree $d$ via semidefinite optimization.

\section{A. PSS approximations of the 6D swept-volume}

The polynomial optimization problem reads:

Problem 3 (Approximate PSS for the swept-volume):

Let the semi-algebraic set $\overline{\mathcal{K}}=\bigcup_{i=1, \ldots, N} \overline{\mathcal{K}}_{i}$ be given by the union of $N$ basic compact semi-algebraic sets $\overline{\mathcal{K}}_{i}$, a given bounding hyper-rectangle $\mathcal{B} \supseteq \overline{\mathcal{K}}$ and also a fixed degree $d$. Solve the optimization problem

$$
\begin{aligned}
& w_{d, \overline{\mathcal{K}}}^{*}=\inf _{p \in \mathbb{R}\left[x_{r}^{0}\right]_{d}}\|p\|_{1}=\int_{\mathbb{B}} p\left(x_{r}^{0}\right) \mathrm{d} x_{r}^{0}, \\
& p \geqslant 0 \text { on } \mathcal{B}, \\
& \text { s.t. } p \geqslant 1 \text { on } \overline{\mathcal{K}}_{1} \text {, } \\
& \text {..., } \\
& p \geqslant 1 \text { on } \overline{\mathcal{K}}_{N} .
\end{aligned}
$$

The main result is the following (its proof is very similar to the one given in [8, Thm. 2]).

Theorem 1: The infimum in Problem (15) is attained for a polynomial $p_{d, \overline{\mathcal{K}}}^{*} \in \mathbb{R}\left[x_{r}^{0}\right]_{d}$. Moreover, $\mathrm{PSS}_{p_{d, \overline{\mathcal{K}}}^{*}} \supseteq \overline{\mathcal{K}}$, $w_{d+1, \overline{\mathcal{K}}}^{*} \leqslant w_{d, \overline{\mathcal{K}}}^{*}$ and $\lim _{d \rightarrow \infty} w_{d, \overline{\mathcal{K}}}^{*}=\operatorname{vol}(\overline{\mathcal{K}})$.

The polynomial $p_{d, \overline{\mathcal{K}}}^{*}$ can be seen as an approximation of the indicator function $1_{\overline{\mathcal{K}}}$ of the set $\overline{\mathcal{K}}$. Such an approximation can be obtained by solving a convex optimization problem whose constraints are Linear Matrix Inequalities (LMIs). Moreover, as the degree of the approximation $d$ increases, the sequence $\left(p_{d}\right)_{d \geqslant 1}$ converges in $\mathcal{L}^{1}$-norm, almost uniformly and almost everywhere to the indicator function of the set $\overline{\mathcal{K}}$ of interest. This can be thought as a direct generalization of classical approximation by ellipsoids. Indeed if degree- 2 PSS approximations are used, we exactly recover wellknown semi-definite optimization-based approaches.

Note that Problem (15) has a dual infinite-dimensional linear problem on measures, namely, denoting the classical Lebesgue measure on $\mathcal{B}$ by $\lambda_{\mathcal{B}}$ :

$$
\begin{aligned}
v_{\overline{\mathcal{K}}}^{*}= & \sup _{\mu_{1}, \ldots, \mu_{N} \in \mathcal{M}(\mathcal{B})} \sum_{i=1}^{N} \mu_{i}\left(\overline{\mathcal{K}}_{i}\right), \\
& \sum_{i=1}^{N} \mu_{i} \leqslant \lambda_{\mathcal{B}}, \\
\text { s.t. } & \operatorname{supp}\left(\mu_{i}\right) \subseteq \overline{\mathcal{K}}_{i}, i=1, \ldots, N \\
& \mu_{i} \geqslant 0, i=1, \ldots, N .
\end{aligned}
$$

In addition, $v_{\overline{\mathcal{K}}}^{*}=\lim _{d \rightarrow \infty} w_{d, \overline{\mathcal{K}}}^{*}=\operatorname{vol}(\overline{\mathcal{K}})[8]$.

Remark 2: It is very interesting to observe that $\mathrm{Pb}$. (13) and (16) are very similar: the main difference resides only in the initial measure considered. For $\mathrm{Pb}$. (13), one aims at computing the integral of a Gaussian measure $\mu_{I}$ to a union of semi-algebraic sets $\mathcal{K}$, while for $\mathrm{Pb}$. (16) the Lebesgue measure $\lambda_{\mathcal{B}}$ is used instead, which simply corresponds to computing the integration of a union of semi-algebraic sets.

We provide in what follows the basic details of the numerical computation of the solution of Problem (15), which is more or less standard in the field of Polynomial Optimization [12].

\section{B. SOS relaxations of Problem (3)}

Recall that the constraints of Problem (3) are:

- the polynomial $p$ is positive on $\mathcal{B}$,

- $p-1$ is positive on $\overline{\mathcal{K}}_{i}, \forall i=1, \cdots, N$.

A common strategy for enforcing positivity is by requiring the polynomial to be sum-of-squares (SOS). Let us denote the convex cone of real polynomials that are SOS by $\Sigma^{2}[x] \subset \mathbb{R}[x]$ and respectively, $\Sigma^{2}[x]_{2 k} \subset$ $\mathbb{R}[x]_{2 k}$, its subcone of SOS polynomials of degree at most $2 k$. Using Putinar's Positivstellensatz [19], [12], Problem (15) becomes, when fixing $\ell \in \mathbb{N}$ :

$$
\begin{aligned}
& w *_{2 \ell, d, \overline{\mathcal{K}}}=\inf _{p \in \mathbb{R}\left[x_{r}^{0}\right]_{d}} \int_{\mathcal{B}} p\left(x_{r}^{0}\right) \mathrm{d} x_{r}^{0}, \\
& \left\{\begin{array}{l}
p-\sigma_{0, \mathcal{B}}-\sum_{1 \leqslant j \leqslant 6} g_{j, \mathcal{B}} \sigma_{j, \mathcal{B}}=0 \\
\sigma_{0, \mathcal{B}} \in \Sigma^{2}\left[x_{0}^{0}\right]_{2 \ell}, \\
\sigma_{j, \mathcal{B}} \in \Sigma^{2}\left[x_{r}^{0}\right]_{2(\ell-1)}, \forall j=1, \cdots, 6,
\end{array}\right. \\
& \left\{\begin{array}{l}
p-\sigma_{0, \mathcal{K}_{1}}-g_{1} \sigma_{1, \mathcal{K}_{1}}-\sum_{1 \leqslant j \leqslant 6} g_{j, \mathcal{B}} \sigma_{1, j, \mathcal{B}}=1 \\
\sigma_{0, \mathcal{K}_{1}} \in \Sigma^{2}\left[x_{r}^{0}\right]_{2 \ell}, \\
\sigma_{1, \mathcal{K}_{1}} \in \Sigma^{2}\left[x_{r}^{0}\right]_{2(\ell-1)}, \\
\sigma_{1, j, \mathcal{B}} \in \Sigma^{2}\left[x_{r}^{0}\right]_{2(\ell-1)}, \quad \forall j=1, \cdots, 6,
\end{array}\right. \\
& \left\{\begin{array}{l}
p-\sigma_{0, \mathcal{K}_{N}}-g_{N} \sigma_{N, \mathcal{K}_{N}}-\sum_{1 \leqslant j \leqslant 6} g_{j, \mathcal{B}} \sigma_{N, j, \mathcal{B}}=1 \\
\sigma_{0, \mathcal{K}_{N}} \in \Sigma^{2}\left[x_{r}^{0}\right]_{2 \ell}, \\
\sigma_{N, \mathcal{K}_{N}} \in \Sigma^{2}\left[x_{r}^{0}\right]_{2(\ell-1)}, \\
\sigma_{N, j, \mathcal{B}} \in \Sigma^{2}\left[x_{r}^{0}\right]_{2(\ell-1)}, \forall j=1, \cdots, 6
\end{array}\right.
\end{aligned}
$$

where the constraints defining the sets $\overline{\mathcal{K}}_{i}$ are:

$$
\begin{aligned}
& \overline{\mathcal{K}}_{i}:=\left\{x_{r}^{0} \in \mathbb{R}^{6}: g_{i}\left(x_{r}^{0}\right) \geqslant 0, g_{j, \mathcal{B}} \geqslant 0, j=1, \ldots, 6\right\}, \\
& i=1, \ldots, N,
\end{aligned}
$$

and the polynomial $g_{i}$ is obtained from Equation (10),

$$
\begin{aligned}
& g_{i}\left(x_{r}^{0}\right):=R^{2}-x_{r}^{0^{T}} \Phi\left(t_{i}, t_{0}\right)^{T} I_{11} \Phi\left(t_{i}, t_{0}\right) x_{r}^{0}, \\
& i=1, \ldots, N,
\end{aligned}
$$

The polynomials $g_{j, \mathcal{B}}$ form the constraints defining the hyper-rectangle $\mathcal{B}$,

$$
g_{j, \mathcal{B}}\left(x_{r}^{0}\right):=\left(x_{r_{j}}^{0}-a_{j}\right)\left(b_{j}-x_{r_{j}}^{0}\right), j=1, \ldots, 6 .
$$

\section{Proposition 3 (Convergence of LMI hierarchy):}

For each fixed $d \in \mathbb{N}$, the value of Problem (17) converges to $w_{d, \overline{\mathcal{K}}}^{*}$, as $\ell \rightarrow \infty$ and moreover, for any $2 \ell \geqslant d$, the solution $p_{2 \ell, d, \overline{\mathcal{K}}}^{*}$ of Problem (17) satisfies the constraints of Problem (15) i.e., $\operatorname{PSS}_{p_{2 \ell, d, \bar{K}}^{*}}$ is a PSS approximation of $\overline{\mathcal{K}}$. 
Proof: The proof can be found in [14].

Let us briefly discuss the simplification obtained when the Gaussian uncertainty on the velocity can be neglected in the six dimensional relative dynamics (position, velocity), during the encounter time interval $\mathcal{T}$.

\section{No velocity uncertainty}

In this simplified setting, the swept-volume is described as a compact union of 3-dimensional ellipsoids according to Proposition 11 Algorithm 1 summarizes the computation of the PSS approximation in this case. Its correctness follows from Propositions 1 and 3 The only technicality (described for completeness in Lines $3-4$ ), resides in computing a bounding box $\mathcal{B}$.

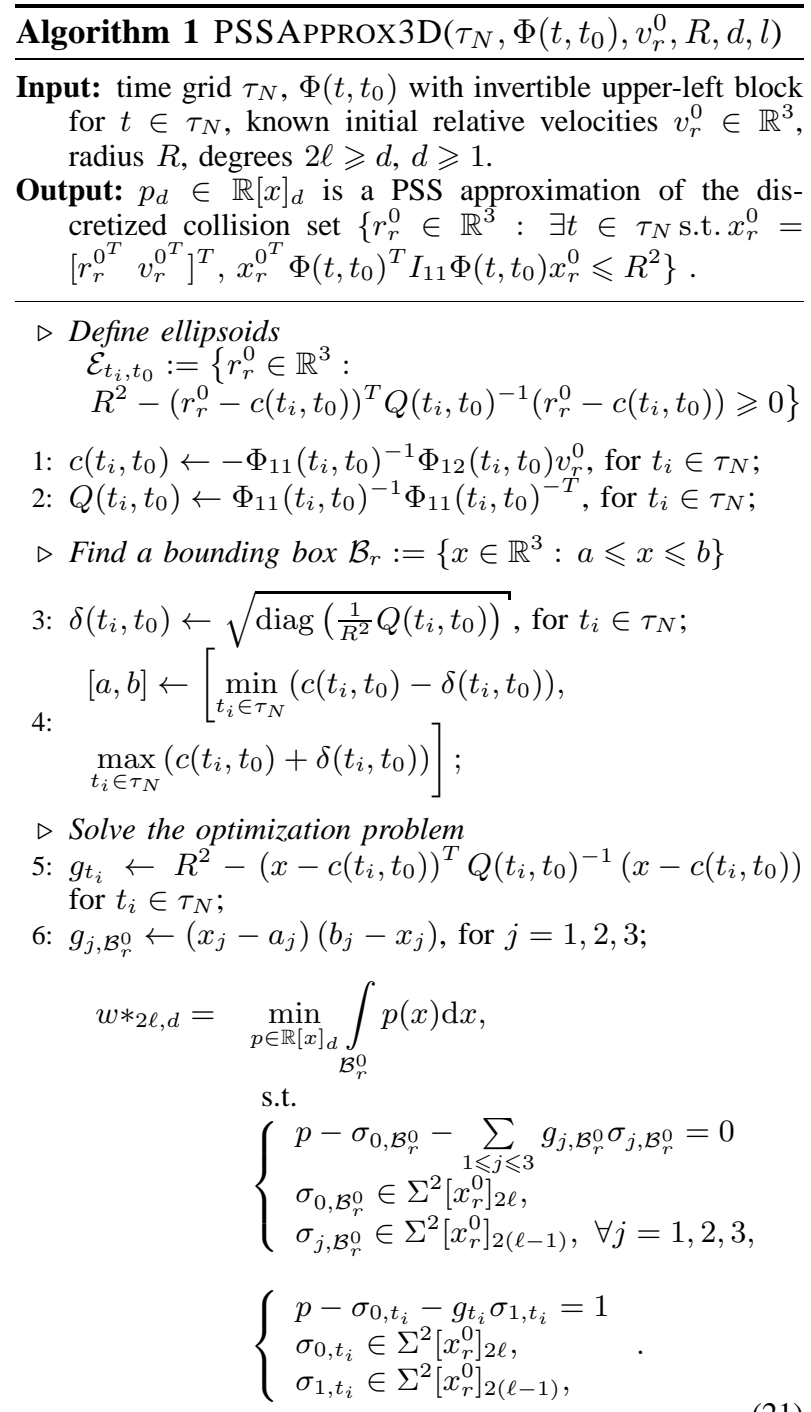

7: return $p_{2 \ell, d}^{*}=\operatorname{argmin}$ 21

For the general 6-dimensional case a similar algorithm can be designed, which solves Problem 17, with the ad- ditional requirement (and approximation) that a compact bounding box needs to be provided as input. Moreover, these algorithms are easily tractable in software.

\section{Implementation Details}

Firstly, the objective function

$$
\int_{\mathcal{B}} p(x) \mathrm{d} x=\sum_{0 \leqslant|i| \leqslant d} p_{i} \int_{\mathcal{B}} x^{i} \mathrm{~d} x,
$$

which is a linear function of the coefficients $p_{i}$ of the polynomial $p$, requires the computation of the Lebesgue moments $m_{i}:=\int_{\mathcal{B}} x^{i} \mathrm{~d} x$, which is straightforward.

Remark 3 (Box scaling): In the implementation, a scaling of $\mathcal{B}$, to the unit box $[-1,1]^{n}$ is important for the numerical quality of the results. Also, in line with Remark 2, from a theoretical perspective, working with the Lebesgue or the Gaussian measure is similar. In practice, computing the Lebesgue moments on $[-1,1]^{n}$, offered the best quality numerical results.

Secondly, the constraints can be recast in terms of Linear Matrix Inequalities (LMIs); this is already a classical strategy and several software tools are available to model problems of the form above, like for instance the Matlab Toolbox YALMIP [15]. Finally, this boils down to solving only a semi-definite programming problem (whenever the degrees $d$ and $l$ are fixed), which was done with the Mosek SDP solver [17].

\section{GAUSSIAN INTEGRATION ON THE SWEPT-VOLUME}

While a compact description of the swept-volume as a PSS is interesting in itself, recall that the complete goal of Problem 2 was the integration of a Gaussian distribution on this swept-volume. To this end, besides the formulation of the optimization Problem (13), we have developed two different strategies that use the obtained PSS directly, and depend upon the assumption on velocity uncertainties:

- The 3D case: Algorithm 1 returns a polynomial $p_{2 \ell, d}^{*}$, which provides an implicit representation of the approximated volume. This is used as input for [20, Algorithm 3], which automatically determines a highorder accurate numerical quadrature for the evaluation of integrals over volumes, whose geometry is defined implicitly via a fixed level set of a smooth function $\phi: \mathbb{R}^{3} \rightarrow \mathbb{R}$. Obviously in our case, $\phi=p_{2 \ell, d}^{*}$.

- The 6D case: Similarly, after Problem 17 is solved for an optimal $p_{2 \ell, d}^{*}$, the integral of a Gaussian distribution over the volume $\operatorname{PSS}_{p_{2 \ell, d}^{*}}$ has to be evaluated. Since the code of [20] is currently restricted to $3 \mathrm{D}$, a basic Monte Carlo sampling is done, which consists in simply checking whether $p_{2 \ell, d}^{*}\left(X_{\mathrm{s}, i}\right) \geqslant 1$ for each sample $X_{\mathrm{s}, i}$. This procedure is to be replaced by a $6 \mathrm{D}$ implementation of the algorithm in [20]. 
Moreover, to cross-check the validity and quality of the proposed methods, a brute-force Monte Carlo process was also designed. Given for completeness in Algorithm 2 2 it uses the very idea of the representation of the swept-volume as a union $\overline{\mathcal{K}}$ of compact sets $\overline{\mathcal{K}}_{i}$. It is simply based on testing whether an initial condition $x_{r}^{0} \in \mathbb{R}^{6}$ leads to a collision in the time interval $\mathcal{T}$, i.e. whether $x_{r}^{0}$ belongs to one of the $\overline{\mathcal{K}}_{i}(i=1, \ldots, N)$.

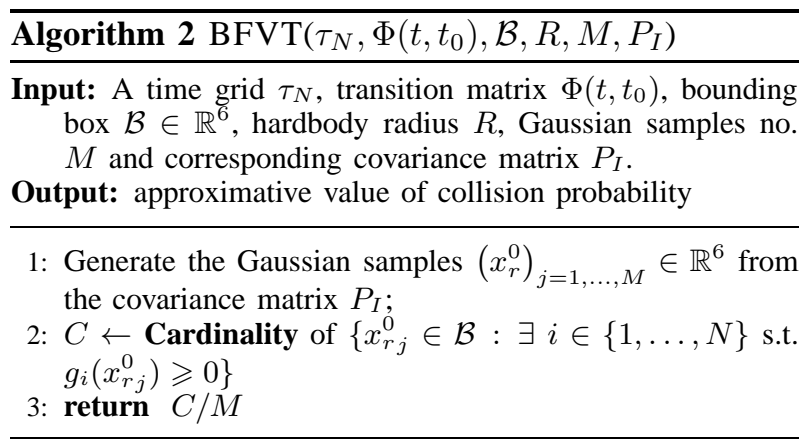

\section{NUMERICAL EXAMPLES}

Two numerical examples borrowed from the paper by S. Alfano [2] are used to illustrate the proposed approach. The Gaussian distribution of both the primary and secondary is known at TCA. A nonlinear two-body Keplerian analytical propagation is used for both the primary and secondary mean vectors, then the norm of the mean relative position (miss distance) is computed on the time interval of the encounter and plotted. The transition matrix $\Phi\left(\cdot, t_{0}\right)$ is computed by performing a linearization of the Keplerian dynamics with respect to the primary object trajectory, following the classical algorithm of Shepperd [22]. For completeness, the Gaussian distribution of the relative state at TCA (with or without velocity uncertainty) is propagated using the transition matrix on the time interval of the encounter. Then, the so-called instantaneous collision probability is plotted for both cases. This indicator is useful in practice since it shows the probability of collision at each given instant. The formula consists in computing a Gaussian integral over a 3D ball and semi-analytical efficient algorithms are available [21]. Finally, the 3D sweptvolume shape and its PSS approximation are depicted.

\section{A. Example 1}

The first example is the case 7 from [2], involving nonlinear relative motion for two satellites in Low Earth Orbits (LEO) where the mean miss distance at TCA is less than the hard-body radius and the relative velocity at TCA is low $(0.19 \mathrm{~m} / \mathrm{s})$. This example was intended to test the sampling methods of the literature, since a low probability of collision is expected and a great number of Monte Carlo samples is needed to get an accurate result. Let us first check the impact of velocity

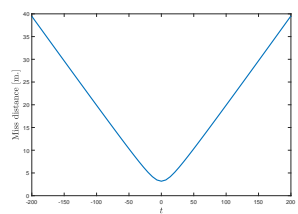

(a)

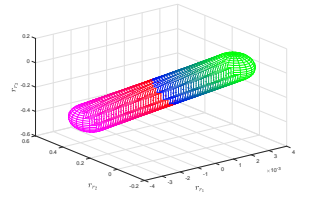

(c)

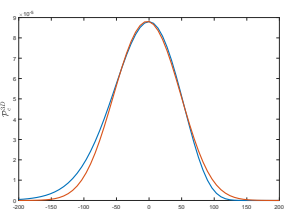

(b)

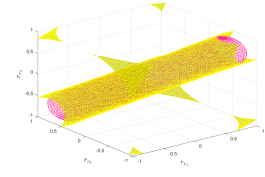

(d)
Fig. 2: (a) Miss distance (m.) (b) Instantaneous probability of collision $\mathcal{P}(t)$ with (blue) or without velocity uncertainties (red) at TCA (c) 3D Swept-volume shape (no velocity uncertainty) (d) PSS approximation of degree $d=4$.

uncertainty at TCA. The instantaneous probability is plotted in Figure 2b, computed with or without relative velocity uncertainty at TCA. Considering the similarity of the two curves, one can consider that the velocity uncertainty at TCA may be neglected in this specific case and the 3D version of Algorithm 1 may be applied. On the time interval $\mathcal{T}=[-1420,1420]$, the exact union of ellipsoids is plotted in Figure 2c with various colors for each time discretization. With $d=2 \ell=4$ this algorithm allows for plotting in Figure 2d the PSS (in a unit cube scaled box). Then, applying the quadrature gives $\tilde{\mathcal{P}}_{c}=0.000165$, while Algorithm 2 with $10^{6}$ samples gives a collision probability of 0.000158 , confirming that we get a good approximation. Note that this result agrees with the ones presented in [2] where the probability is evaluated as 0.000161 for a highly computationally demanding Monte Carlo approach (662 million Monte Carlo runs needed) and 0.000164 for the voxels method. Finally, the results are consistent with Coppola's formulation when assuming no velocity uncertainty (formula (40) of [7]) which gives 0.000159.

\section{B. Example 2}

We consider the encounter [2, Example No. 9], of two objects in highly-eccentric orbits (HEO) where the mean miss distance at TCA is greater than the combined object radius. The computed instantaneous collision probability is given in Figure 3 (b) and (c) with/without relative velocity uncertainty considered at TCA.

Firstly, our method is applied assuming that there is no velocity uncertainty (the 3D case). In Figure 3(d), the exact swept-volume (union of 40 ellipsoids obtained on the time interval $\mathcal{T}=[-10800,10800])$ is plotted. Algorithm 11 applied with $d=2 l=8$, requires about 10 seconds (Matlab execution on a classical workstation) for obtaining the degree-8 PSS, plotted in Figure 3(e). 
Finally, the code of [20, Algorithm 3] requires less than 10 seconds to evaluate the Gaussian integral over the PSS computed before, leading to $\tilde{\mathcal{P}}_{c}=0.2825$, which is confirmed by brute-force simulations. Formula (40) in [7] gives 0.2715 .

As observed in Figure 3(b) and (c) and also confirmed by the results in [2], the relative velocity uncertainty does matter in this case: Algorithm 2 provides a value similar to that of [2], of 0.36511 . Our 6D method, with $d=2 l=8$, provides a PSS volume, whose projection in $3 \mathrm{D}$, for the fixed mean velocity value at TCA is plotted in Figure 3 (e). One observes an overestimation for this case. Depending on the $h-\sigma$ bounding ellipsoid considered as bounding, the results obtained with our method range from 0.50 for $6-\sigma$ to 0.371 for $1-\sigma$.

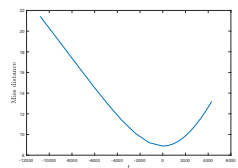

(a)

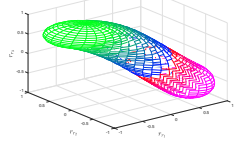

(d)

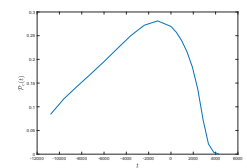

(b)

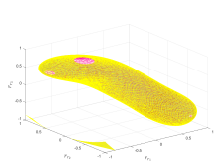

(e)

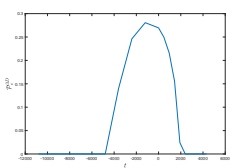

(c)

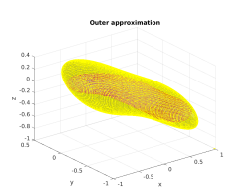

(f)
Fig. 3: (a) Miss distance (m) (b) Instantaneous collision probability $\mathcal{P}(t)$ with (c) and without (d) velocity uncertainty (d) 3D Swept-volume shape (no velocity uncertainty) (e) PSS approximation $d=8$ (f) Projection of 6D PSS approx, $d=8$, for a fixed mean velocity.

\section{CONCLUSION}

By using models from polynomial optimization, we have provided (i) a theoretical framework and (ii), practical algorithms to obtain approximate closed-form descriptions of the set of collision-prone states (and an approximation of their Gaussian measure). This allows efficient approximations of non-convex shapes which can be used effectively for long-term and repeated conjunctions, generalizing both Chan's and Coppola's formulations. We are confident that visual accurate outer-approximation of the swept volume can provide further insight on classifying the types of encounter occurring in practice. Along these lines, a further complexity and numerical analysis on the provided algorithms would help to better assess and design more tuned and efficient algorithms for special practical cases.

\section{REFERENCES}

[1] M.R. Akella and K.T. Alfriend. Probability of collision between space objects. Journal of Guidance, Control and Dynamics, 23(5):769-772, 2000.
[2] S. Alfano. Satellite conjunction Monte Carlo analysis. Advances in the Astronautical Sciences, 134:2007-2024, jan 2009.

[3] D. Arzelier, F. Bréhard, M. Joldeş, J.B. Lasserre, L. Martire, and A. Rondepierre. Global probability of collision: problem modelling via occupation measure. Technical Note AJ/AR/EO2016-6457, LAAS-CNRS, October 2017.

[4] F.K. Chan. Spacecraft Collision Probability. American Institute of Aeronautics and Astronautics, 2008.

[5] F.K. Chan. Hovering collision probability. In AAS/AIAA Space Flight Mechanics Meeting, number AAS 15-234, Williamsburg, VA, USA, January 2015.

[6] K. Chan. Spacecraft collision probability for long-term encounters. Number AAS 03-549, Big Sky, Montana, USA, 2003.

[7] V.T. Coppola. Including Velocity Uncertainty in the Probability of Collision between Space Objects. Advances in the Astronautical Sciences, 143, 2012.

[8] F. Dabbene, D. Henrion, and C.M. Lagoa. Simple approximations of semialgebraic sets and their applications to control. Automatica, 78:110-118, 2017.

[9] J. L. Foster and S. E. Herbert. A Parametric Analysis of orbital Debris Collision Probability and Maneuver Rate for Space Vehicles. Technical report, NASA Johnson Space Center, August 1992.

[10] D. Henrion, J.B. Lasserre, and C. Savorgnan. Approximate volume and integration for basic semialgebraic sets. SIAM review, 51(4):722-743, 2009.

[11] M. Korda. Moment-sum-of-squares hierarchies for set approximation and optimal control. PhD thesis, École Polytechnique FéDérale de Lausanne, 2016.

[12] J.B. Lasserre. An Introduction to Polynomial and Semi-Algebraic Optimization. Cambridge University Press, 2015.

[13] J.B. Lasserre. Computing gaussian and exponential measures of semi-algebraic sets. Advances in Applied Mathematics, 91:137163, October 2017.

[14] J.B. Lasserre and Y. Emin. Semidefinite relaxations for lebesgue and gaussian measures of unions of basic semialgebraic sets. Mathematics of Operations Research, 44(4):1477-1493, 2019.

[15] J. Lofberg. YALMIP : a toolbox for modeling and optimization in MATLAB, 2004. http://yalmip.github.io/

[16] D.P. McKinley. Development of a nonlinear probability of collision tool for the Earth observing system. In AIAA/AAS Astrodynamics Specialist Conference and Exhibit, number AAS2006-6295, August 2006.

[17] MOSEK ApS. MOSEK Version 8.0.0.64, April 2017. https://www.mosek.com/products/mosek

[18] R.P. Patera. Satellite collision probability for nonlinear relative motion. Journal of Guidance Control and Dynamics, 26(5):728733, 2003.

[19] M. Putinar. Positive polynomials on compact semi-algebraic sets. Indiana University Mathematics Journal, 42(3):969-984, 1993.

[20] R.I. Saye. High-order quadrature methods for implicitly defined surfaces and volumes in hyperrectangles. SIAM Journal on Scientific Computing, 37(2):A993-A1019, 2015.

[21] R. Serra, D. Arzelier, M.M. Joldes, J.B. Lasserre, A. Rondepierre, and B. Salvy. Fast and accurate computation of orbital collision probability for short-term encounters. Journal of Guidance Control and Dynamics, 2016.

[22] W.S. Shepperd. Universal Keplerian State Transition Matrix. Celestial Mechanics, 35:129-144, 1985.

[23] S. Streif, D. Henrion, and R. Findeisen. Probabilistic and set-based model invalidation and estimation using lmis. IFAC Proceedings Volumes, 47(3):4110-4115, 2014. 